\title{
Consent to treatment and clinical decision analysis
}

\author{
A solution to medical uncertainty and public doubt?
}

\author{
ERnest P. Worrall, Consultant Psychiatrist, Southern General Hospital, Glasgow
}

The 1984 Scottish Mental Health Act (and its counterpart in England and Wales) invoked unique restrictions in medical practice in this country. For the first time certain standard treatments could not be given to particular patients unless an independent second opinion doctor authorised that treatment. Fortunately, in respect of drug treatment and ECT the second opinion doctors are themselves practising clinicians. Second opinion doctors are asked to give their opinion about the suitability of a proposed treatment using the following guidelines: "the appointed doctor will have in mind his/her understanding of practice accepted as proper by a responsible body of medical men skilled in this particular art in Scotland at this time and should avoid any idiosyncratic view of treatment however firmly held".

That is rather a mouthful and I think could be fairly summarised as meaning 'normal accepted practice'. What is normal accepted practice? There will be inevitable differences in normal accepted practice between groups of doctors, e.g. the widely quoted threefold difference in ECT rates between Yorkshire and Oxford in 1979 (Pippard \& Ellam, 1981). This is not reassuring to patients but is not unique to psychiatry. An example of a patient's anguish at belatedly discovering the wide difference between two consultants' views of accepted practice in treating carcinoma of the breast is well described by Angela Prior (1987) (a patient with carcinoma of the breast) in a Personal View in the British Medical Journal. She made the following plea-probably a naive one in the eyes of most doctors - "approaches to breast cancer should not vary so much from one hospital to another, nor should treatment be dependent on the opinion of one surgeon. It should not be, as one doctor put it, 'a kind of lucky dip' ". In psychiatry, in conjunction with the new Act, an early draft Code of Practice was mooted and published but was not welcomed by most psychiatrists as the draft was considered to be over-complicated and unrealistic. The subsequent final Code of Practice went to the opposite extreme and was entirely bland. Codes of Practice therefore seem not to be capable of resolving any potential uncertainty that might occur between a referring psychiatrist and a second opinion doctor about what is normal accepted practice.
Normal accepted practice is imprecise and incapable of other than arbitrary definition. Doctors would be unable to agree on the limits of any arbitrary definition and would quite rightly oppose this. It is, in fact, the flexibility inherent in the imprecision of the idea that makes it attractive to doctors involved in consent to treatment decisions, but it is the range of this flexibility which might partly explain the public's lack of reassurance and, if they are honest, doctors' potential uncertainty. Two psychiatrists recently wrote diametrically opposing views about the original English draft Code of Practice. One was a psychiatrist in practice at Broadmoor who supported the concept (Grounds, 1986). The other was Dr John Hamilton, Physician Superintendent at Broadmoor, who wrote a critical leading article about the Code (Hamilton, 1986). Although doctors could readily understand these disagreements they can only reinforce the doubts of those members of the public who are suspicious about our practice: if two psychiatrists working in the same hospital cannot even agree about the best way to go about a problem the public might think they are even less likely to be in agreement about what should be done in actual clinical practice. The problem in a nutshell remains that doctors think they know best and some of the public do not trust them.

One possible answer to this difficulty might be to make explicit the decision-making process by using the techniques of decision analysis. These are employed in business management when the best decision between possible options is unclear. It is a method of making explicit all the possible outcomes of different decisions, assigning a probability level to each of the outcomes and then, when necessary, quantifying the values attached to these outcomes to allow the best decision to be made. The only difference between this and good intuitive clinical decision-making is the explicitness of the various steps in formal decision analysis. A description of the technique applied to medical practice can be found in Weinstein et al (1980) and more accessibly in a paper by Pauker \& Kassirer (1987). In medicine the process has been applied to decisions which pose more than usual difficulty but one of the basic tools in the technique - the decision tree-might provide a 
helpful frame of reference in any potential debate between a referring clinician and a second opinion doctor. The main advantage this would have over codes of practice is that the technique is not restrictive and take account of the individual circumstances of a particular patient and referring clinician. Because the process is explicit it might reassure patients and those concerned members of the public who are open to rational argument.

Clinical decision analysis takes account of published research findings, clinicians' own estimates of probability and if need be patient's preferences (although the outcome values may then be more difficult to quantify). The main difference in constructing a clinical decision tree for treatment options and just trying to reach a consensus view about published trials, or even a meta-analysis of such trials, lies in decision trees taking into account much more information than most published trials consider. Controlled trials are answering more specific and more limited questions than those facing the clinician who has to ask himself "For patient John Smith with an X probability of disease A complicated by associated conditions $B$ and $C$, what is the best treatment between $D$ and $E$ or neither of these two now but treatment $F$ at a later time?". Retrospective trials, although more naturalistic, rarely take into account withdrawal from treatment.
The decision tree shown in Fig. 1 is an illustration of decision analysis applied to a particular psychiatric problem which has relevance for consent to treatment. This decision tree considers the options for treating a unipolar depressed patient with delusions. This example was chosen for the following reasons. It is unlikely such a patient could give valid consent for ECT and since that is one treatment option, second opinion authorisation may be required. There is debate about the treatment of first choice for such a patient and there is sufficient literature available to make estimates of the probability of the various outcomes with different treatments. Lastly, this decision tree is actually used by me in my own clinical practice, used as an example in training junior staff and the social worker who is attached to my team and who is a Mental Health Officer involved in consent to admission and understands the model and takes a valued part in decisions about treatment decisions using the model. This is important as a decision to detain the patient and therefore give consent to admission may depend on the nature and likely outcome of the treatment proposed. If that treatment is ECT the Royal College of Psychiatrists has advised that when an informal patient is unable to give valid consent to ECT the patient should be detained and second opinion authorisation for ECT obtained.

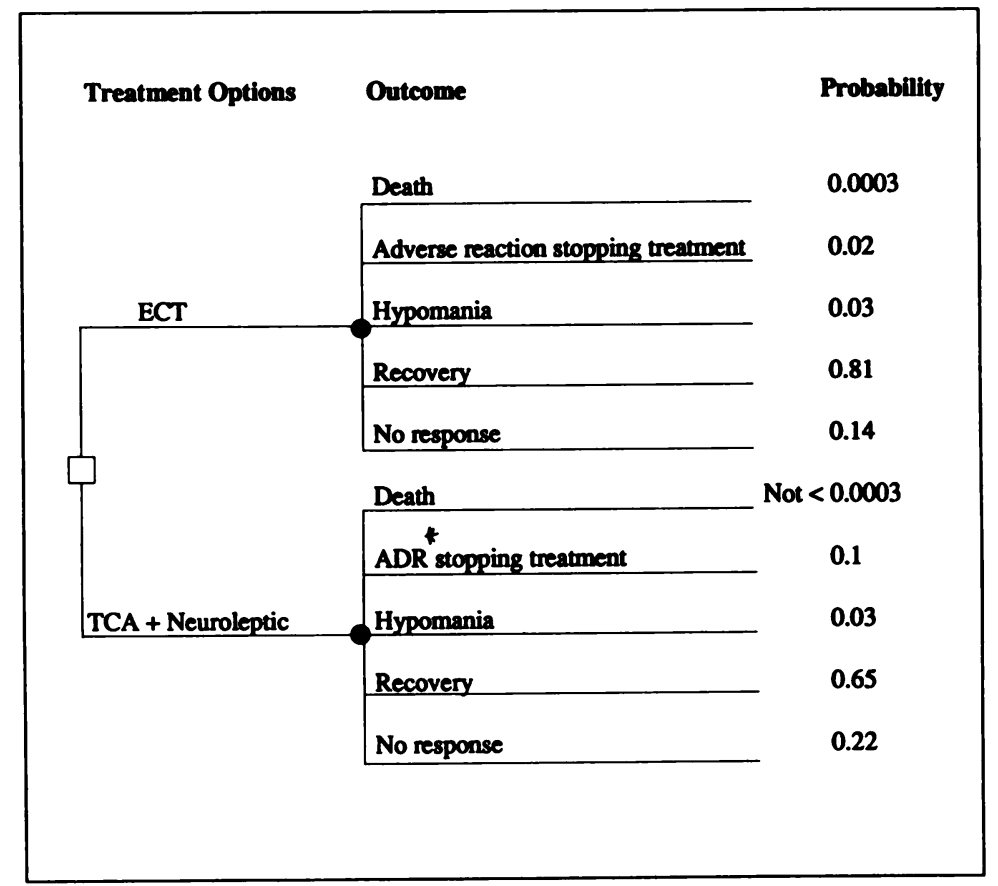

Fig. 1. Decision tree for treatment of unipolar depressed patient with delusions. *ADR: Adverse drug reaction. 


\section{Explanation of the outcome probabilities}

The decision tree considers all the possible outcomes with ECT and with combined tricyclic antidepressant/neuroleptic (TA/N) drug treatment. (The references for the probability figures for each of the outcomes is available from the author). In the main the figures for "adverse reactions stopping treatment" and development of hypomania with ECT were obtained from pooling the figures from four British controlled trials of ECT in depressive illness. The probability figure for "adverse drug reactions stopping treatment" with a TA/N combination was obtained from published drug monitoring studies and a prospective trial of TA/N treatment. The figure for hypomania after TA/N treatment was obtained by considering the average figure for the development of hypomania in unipolar depression after tricyclic treatment in the review paper by Wehr \& Goodwin (1987) and then reducing that figure by two-thirds to allow for the concomitant administration of a neuroleptic, which should have some preventive action. The probability of "recovery" and of "no response" was obtained by taking the figures for both these outcomes from prospective and retrospective trials and then applying those figures to the residual patients after attrition by "adverse drug reactions stopping treatment" or hypomania. It could be argued that hypomania, especially if only mild, as is likely with both sorts of treatments, could be considered recovery but even if that were done it would not alter the relative proportion of recovered patients with both sorts of treatment. A third option - tricyclic treatment alone - is not a reasonable option in this situation as numerous trials have shown that treatment in deluded unipolar depressed patients only produce recovery in about $30 \%$ of such patients.

\section{Discussion}

This tree is simple to use and on every outcome ECT is the best option and therefore values for outcomes do not have to be further quantified. In actual use for individual patients, particular characteristics of the patient will alter some of the probability estimates. For example, if the patient has made a full recovery with ECT in previous episodes of illness the probability of recovery if the patient completes treatment will have to be raised. If the patient has previously responded to drug treatment then the probability of recovery would also need to be raised but, in the author's estimate, less than the rise in probability increase in the case of previous ECT. These latter types of estimates of probability-response to ECT if the patient has previously responded and the equivalent estimate for drug treatment-are examples of subjective probability. The literature does not give sufficient information to calculate an observed probability and the doctor has to estimate this from his own clinical experience and from discussion with colleagues. Making estimates of subjective probability is a crucial part of decision analysis. The argument for doing this is that doctors already do this intuitively and in keeping with the explicitness in decision-making analysis the doctor must be explicit in assigning a subjective probability element to these events even when the literature does not provide observed probability figures.

Weinstein et al (1980) have described the advantages of decision analysis in resolving treatment controversies for particular patients. It is worthwhile considering the value of this approach in any potential dispute over consent to treatment and second opinions in psychiatric patients. If the two doctors concerned are in disagreement over the proposed treatment the decision tree allows them to be clear over what it is they disagree. There is no hidden agenda for the disagreement. Does one of them think the structure of the tree is inaccurate? Are there other options for management which have not been included? Are there other chance events which should be shown in the tree? Do they differ over probability estimates? If they do, what limits in the estimates would they each accept? Could they agree which papers from the literature they would accept for the probability estimates? Do they disagree on the value attached to different outcomes?

Such an approach might not only clarify the situation for doctors in dispute but might convince the public that for psychiatric patients who are either unable to understand the treatment or do not agree to it that, unlike the patient with carcinoma of the breast, treatment was not "a kind of lucky dip".

\section{References}

Grounds, A. (1986) Psychiatry and patients' rights. British Journal of Hospital Medicine, 36, 147-148.

Hamilton, J. (1986) Editorial, British Medical Journal, 292, 1219.

PAuker, S. G. \& Kassirer, J. P. (1987) Medical progress: decision analysis. New England Journal of Medicine, 316, 250-258.

PipPaRd, J. \& Ellam, L. (1981). Electro-convulsive therapy in Great Britain. British Journal of Psychiatry, 139, 563-568.

Prior, A. (1987) Personal View. British Medical Journal, 295, 920.

WEHR, T. S. \& GOODWIN, F. K. (1987) Can antidepressants cause mania and worsen the course of affective illness? American Journal of Psychiatry, 144, 1403-1411.

Weinstein, M. C., Fineberg, H. V. \& Elstein, A. S. (1980) Clinical Decision Analysis. Philadelphia: W. B. Saunders. 